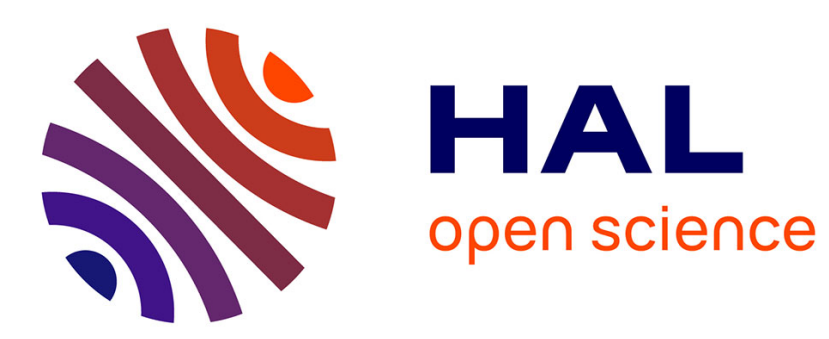

\title{
SPARSE-CODING ADAPTED TO SAR IMAGES WITH AN APPLICATION TO DESPECKLING
}

\author{
Sonia Tabti, Luisa Verdoliva, Giovanni Poggi
}

\section{To cite this version:}

Sonia Tabti, Luisa Verdoliva, Giovanni Poggi. SPARSE-CODING ADAPTED TO SAR IMAGES WITH AN APPLICATION TO DESPECKLING. 2017. hal-01647163v2

\section{HAL Id: hal-01647163 \\ https://hal.science/hal-01647163v2}

Preprint submitted on 6 Dec 2017

HAL is a multi-disciplinary open access archive for the deposit and dissemination of scientific research documents, whether they are published or not. The documents may come from teaching and research institutions in France or abroad, or from public or private research centers.
L'archive ouverte pluridisciplinaire HAL, est destinée au dépôt et à la diffusion de documents scientifiques de niveau recherche, publiés ou non, émanant des établissements d'enseignement et de recherche français ou étrangers, des laboratoires publics ou privés. 


\section{SPARSE-CODING ADAPTED TO SAR IMAGES WITH AN APPLICATION TO DESPECKLING}

\author{
Sonia Tabti * \\ Normandie Univ, ENSICAEN, CNRS, GREYC, \\ 6 Bd du Marchal Juin, Caen, 14000, France
}

\author{
Luisa Verdoliva, Giovanni Poggi
}

DIETI, Università Federico II di Napoli,
via Claudio 21, 80125, Napoli, Italy

\begin{abstract}
In this paper, we propose a sparsity-based despeckling approach. The first main contribution of this work is the elaboration of a sparse-coding algorithm adapted to the statistics of SAR images. In fact, most sparse-coding algorithms for SAR data apply a logarithmic transform to data, so as to convert the noise from multiplicative to additive. Then, a Gaussian prior is adopted. However, using a more suitable prior for SAR data avoids introducing artifacts. The second main contribution proposed is to predict the optimal sparsity degree for each patch based on local image features. Experiments show that this strategy improves upon traditional sparse coding with a low-error-rate stopping criterion.
\end{abstract}

Index Terms - Patches, sparse-coding, coefficient of variation, despeckling, SAR images.

\section{CONTEXT AND MOTIVATION}

The aim of this work is to elaborate a despeckling algorithm using a sparsity-based approach dedicated to SAR images. Because of the peculiar imaging mechanism of SAR systems, images are affected by intense noise. For intensity SAR images, this can be modeled according to Goodman's model [1] by a multiplicative noise following a gamma distribution. The speckle of a SAR image can be attenuated by producing a multi-look image with a lower resolution. To do so, we compute for each pixel the mean of its $L$ neighboring pixels, or if it is a multitemporal image, a temporal mean is computed. The number $L$ is referred to as the number of looks.

The principle of a sparsity-based approach is to approximate a small window (of typical size $8 \times 8$ pixels) extracted from an image, called a patch, by a sparse linear combination of atoms which are the elements of a dictionary $\boldsymbol{D}$ as expressed by the next equation:

$$
\min _{\boldsymbol{u}_{i}}\left\|\boldsymbol{D} \boldsymbol{u}_{i}-\boldsymbol{x}_{i}\right\|_{2}^{2} \quad \text { s.t } \phi\left(\boldsymbol{u}_{i}\right) \text { is sparse }
$$

*Work performed during the post-doc of the first author at the DIETI, Federico II University in Naples. where $\boldsymbol{x}_{i}$ is a patch, $\boldsymbol{u}_{i}$ is the corresponding vector of sparse coefficients, and $\phi$ is the sparse regularization term.

A standard dictionary-based approach, eg. the K-SVD (Singular Value Decomposition) algorithm [2], is divided in two steps repeated until a stopping criterion is satisfied. The first step is the sparse-coding, ie. the computation of the coefficients of the sparse linear combination which depends on the choice of the prior $\phi$. In this algorithm, the prior used is the non-convex $\ell_{0}$ pseudo-norm, $\phi(\boldsymbol{u})=\|\boldsymbol{u}\|_{0}$, which counts the number of non-zero elements in vector $\boldsymbol{u}$ called: the sparsity degree. One can use greedy approaches in order to approximate the solution of this NP-hard problem, the OMP (Orthogonal Matching Pursuit) [3] procedure is often used for this purpose. The second step of a dictionary-based approach is the update of the elements of the dictionary taking the first step into account. Many sparsity-based despeckling methods have been proposed in the past. In [4] and [5] the K-SVD and a K-Means like algorithms are adapted to SAR data respectively. In [6], a PCA (Principal Component Analysis) dictionary is computed and the prior used is an $\ell_{1}$ norm. The authors performed a classification of the patches in two labels: homogeneous and heterogeneous in order to process them separately.

In this work, we suppose that the dictionary is already learned on logarithmically transformed patches extracted from multilook SAR images with K-SVD in order to simplify the optimization problem we will solve. Consequently, we will only focus on the sparse coding step. In Section 2, we will develop a SAR-adapted sparse coding algorithm. In Section 3. we will explain how to compute a map of the estimated sparsity degree for each patch in the image. In Section 4, a detailed description of the proposed algorithm is given. In Section 5 we evaluate how introducing this map improves the results in comparison with a standard sparsity-based despeckling method.

\section{PROPOSED SAR SPARSE-CODING PROCEDURE}

In order to process properly SAR images, despeckling methods should be adapted to the statistics of speckle. In 
this section, we propose a sparse-coding procedure adapted to logarithmically-transformed SAR data. Hence we use a Fisher-Tippett distribution as data-fidelity term which corresponds to the logarithm of the gamma distribution often used to model intensity SAR images. The whole sparse-coding problem is expressed by the next equation:

$$
\{\hat{\boldsymbol{x}}, \hat{\boldsymbol{u}}\}=\underset{\boldsymbol{u}, \boldsymbol{x}}{\operatorname{argmin}} \sum_{i=1}^{N} \lambda\left(x_{i}-y_{i}+\exp \left(y_{i}-x_{i}\right)\right)+\mu\left\|\boldsymbol{u}_{i}\right\|_{0}
$$

where $\boldsymbol{x}$ is the image to recover, $\hat{\boldsymbol{x}}_{i}=\boldsymbol{D} \hat{\boldsymbol{u}}_{i}$ the $i$-th patch of the restored image $\hat{\boldsymbol{x}}, \boldsymbol{y}$ the speckled image, $x_{i}, y_{i}$ are pixels extracted from $\boldsymbol{x}, \boldsymbol{y}$ respectively at position $i, \lambda$ and $\mu$ are regularization parameters and $N$ is the number of pixels.

This optimization problem is difficult to solve. A possible strategy is to use an iterative approach called half-quadratic splitting, as it is proposed for instance in [7] with a Gaussian Mixture Models prior (which was adapted to SAR despeckling in [8]) and in [9] with a sparse prior. It implies to introduce for each patch an auxilairy variable $\boldsymbol{z}_{i}=\boldsymbol{D} \boldsymbol{u}_{i}$ which will allow us to optimize the following function alternatively along $\boldsymbol{x}$ and $\boldsymbol{z}_{i}$ (see [8] for more details):

$$
\begin{array}{r}
\{\hat{\boldsymbol{x}}, \hat{\boldsymbol{u}}\}=\underset{\boldsymbol{x}}{\operatorname{argmin}}\left(\sum_{i=1}^{N} \lambda\left(x_{i}-y_{i}+\exp \left(y_{i}-x_{i}\right)\right)\right. \\
\left.+\min _{\boldsymbol{z}_{i}, \boldsymbol{u}}\left(\frac{\delta}{2} c_{i}\left(x_{i}-\bar{z}_{i}\right)^{2}+\mu\left\|\boldsymbol{u}_{i}\right\|_{0}\right)\right)
\end{array}
$$

where $\boldsymbol{R}_{i}$ is the extracting operator of the $i$-th patch in the image, $\overline{\boldsymbol{z}}=\operatorname{diag}(\boldsymbol{c})^{-1} \sum_{i} \boldsymbol{R}_{i}^{t} \boldsymbol{z}_{i}$ is the uniform reprojection of the patches $\boldsymbol{z}_{i}$ in the image domain, and $c_{i}$ is the number of patches $\boldsymbol{R}_{i} \boldsymbol{x}$ projecting on pixel $i$. Hence, solving problem (3) along $z_{i}$ can be performed by OMP and solving it along $\boldsymbol{x}$ boils down to using the Newton method (10 iterations are enough) as described for the $(t+1)^{\text {st }}$ iteration as:

$$
x_{i}^{(t+1)}=x_{i}^{(t)}-\frac{\left.\lambda\left(1-e^{y_{i}-x_{i}^{(t)}}\right)+\delta c_{i}\left(x_{i}^{(t)}-\bar{z}_{i}\right)\right)}{\lambda e^{y_{i}-x_{i}^{(t)}}+\delta c_{i}}
$$

In theory, as $\delta \rightarrow \infty$ the algorithm converges. In practice, a few iterations (three or four) are enough to obtain a good solution. Algorithm 1 describes the whole SAR-sparse-coding (SAR-SC) procedure proposed. The values of $\delta$ are increasing from one iteration to another. The variance of the FisherTippett distribution, $\psi(1, L)$, refers to the second logarithmic derivative of the gamma function.

\section{COMPUTATION OF THE SPARSITY MAP}

In this section, we explain how to compute the Sparsity Degree Map (SDM) based on a Coefficient of Variation Map (CVM). We first recall that the coefficient of variation of a

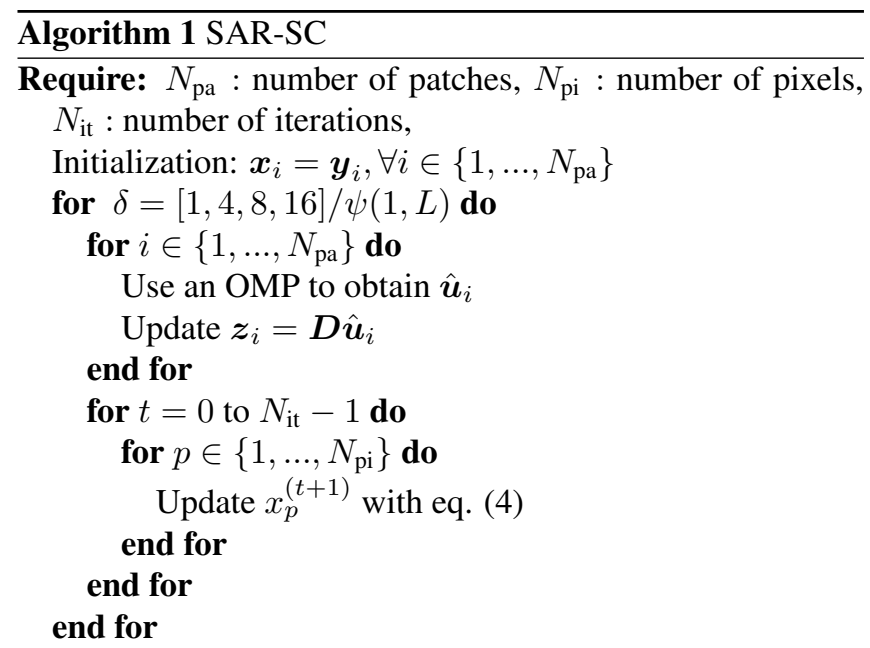

patch $\boldsymbol{x}$ (of size $8 \times 8$ in our setting) is defined as:

$$
\mathcal{C}_{v}(\boldsymbol{x})=\frac{\sigma_{\boldsymbol{x}}}{\mu_{\boldsymbol{x}}}
$$

where $\sigma_{\boldsymbol{x}}$ is the standard deviation of the patch $\boldsymbol{x}$ and $\mu_{\boldsymbol{x}}$ is the mean of $\boldsymbol{x}$. It is widely used in SAR imagery, for classification purpose for instance, since it measures the heterogeneity of a region: the higher the value of the coefficient, the more heterogeneous is the region. We obtain a CVM by computing the coefficient of variation of each patch in the image. To ensure the smoothness of the map, it is better to pre-filter the noisy image before computing the CVM. In this study, the pre-filtering is performed by sparse approximation of each patch in the image using an OMP with a fixed sparsity degree, then averaging the patches to obtain the whole image. Various sparsity degrees have been tested, eventually, we selected $k=1$ as this reduces computation time without any significant impairment of quality. The whole procedure of the sparsity degree map computation is described in the next steps:

- Computation of the CVM on the pre-filtered image.

- Classification of the CVM using K-Means algorithm with $k_{\max }$ labels (meaning that the sparsity degree ranges from 1 to $\left.k_{\max }\right)$. After experimentation, the value of $k_{\max }$ was set to 4 .

- Sorting of the CV centroids for increasing value, and association with sparsity degrees from 1 to $k_{\max }$.

An example of SDM is shown in figure 2

\section{ALGORITHM DESCRIPTION}

\subsection{About the dictionary}

The dictionary used in the proposed approach is learned offline with the K-SVD algortihm applied on logarithmically 


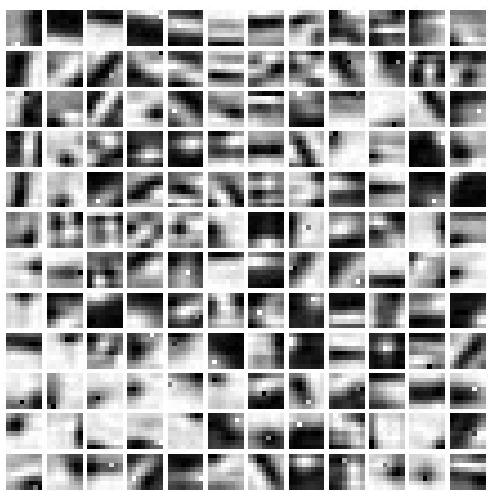

Fig. 1. K-SVD dictionary $(144$ atoms of size $8 \times 8)$ learned on logarithmically-transformed SAR data.

transformed SAR data (Homomorphic K-SVD). The data-set is composed of 8 multi-look SAR images of different size ranging from $200 \times 200$ to $500 \times 500$. The parameters of the algorithm are the same as in the original paper except that, first, the noise level is adapted to SAR data and the number of looks, second, a debiasing step is applied [10]. Consequently, we didn't set a fixed sparsity degree in the OMP and the stopping-criterion was a low error rate. The initialization is a DCT (Discrete Cosine Transform) dictionary. The dictionary obtained is shown in figure 1. It is satisfying because the algorithm managed to capture targets, which are important features in SAR data, and edges with various orientations.

\subsection{Main steps of the proposed algorithm}

The next steps summarize the proposed despeckling algorithm:

- A pilot despeckled image is obtained to compute the CVM. To this end, the despeckling procedure described in section 2 is applied with a sparsity degree $k=1$.

- The SDM is computed as described in section 3

- The SDM and the proposed sparse-coding procedure are used to despeckle each patch with the prescribed sparsity degree in the map.

\subsection{Improving despeckling results in homogeneous areas}

As we can observe in figure 3 , homogeneous areas need more smoothing. To tackle this issue, we propose to represent the patches with a sparsity degree equals to one in the SDM (which are homogeneous as we can observe in the map, fig 2) with a specific small dictionary composed of constant atoms. We set experimentally the number of atoms to 12 with values ranging from 20 to 160 . Figure 3 shows that edges and backscatterring targets are well preseved and homogeneous areas are smoother than in the result without improvement.

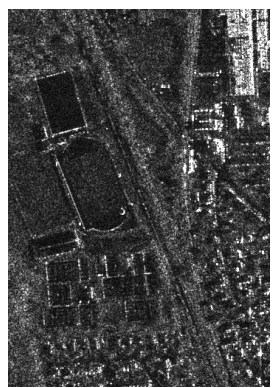

SAR image

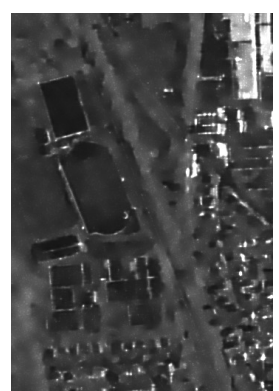

Proposed approach

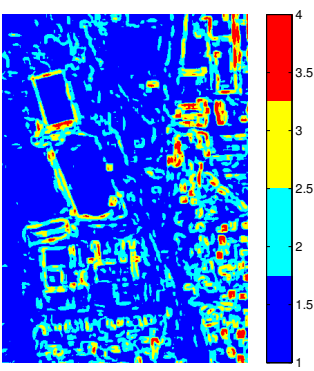

Sparsity map
Fig. 2. Despeckling of the Rosen 3 TerraSAR-X (Infoterra $\mathrm{GmbH}$ ) image and coresponding color-coded SDM.

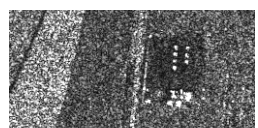

SAR image

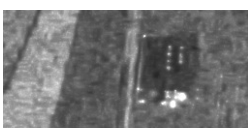

Initial result

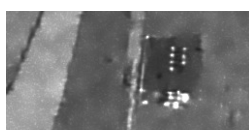

Improved
Fig. 3. Improvment of our results in the homogeneous areas.

\section{RESULTS AND DISCUSSION}

\subsection{Comparison with low error rate sparse approxima- tion}

In the Gaussian noise case, in sparsity-based denoising algorithms, it is usually better to use a sparse-coding algorithm with a low error rate stopping criterion in comparison with one fixed sparsity degree for all the patches or the use of our SDM. This rate is defined by the quadratic difference between the patches and their sparse approximation. In this paragraph, we investigate if this assertion remains true in the gamma noise case. Figure 4 shows results on a natural image with synthetic 2-looks speckle noise. It allows us to compare quantitatively the sparse-coding approach adapated to SAR data with Low-Error-Rate (LER) stopping criterion and with the SDM. We observe that the LER result is blurrier than the one obtained with the proposed approach. The obtained PSNR value confirms this. It should be noted that these observations remain true on real SAR images and the computation time is shorter with the porposed approach. A possible justification is that, since the dynamic range of SAR images is high in comparison with natural images, the error is high too, hence the LER sparse-coding reaches the desired precision slowly.

\subsection{Comparisons with state of the art methods}

In figure 5. we compare our approach with some state of the art methods: 1) Probabilistic Patch-Based (PPB) filter [11], which is the adaptation of the Non-Local means algorithm [12], and has been improved in [13], 2) SAR-BM3D [14] which is the adaptation of the Block-Matching 3D algorithm [15], 3) FANS (Fast Adaptive Non-Local SAR de- 


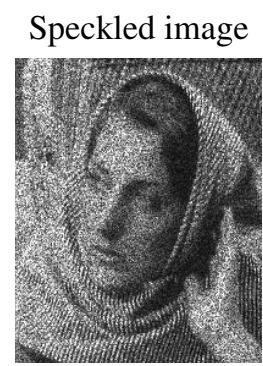

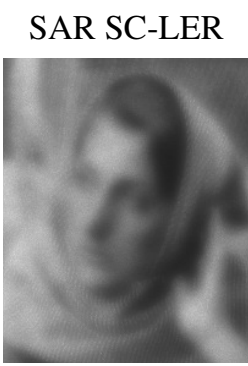

$21.40 \mathrm{~dB}$

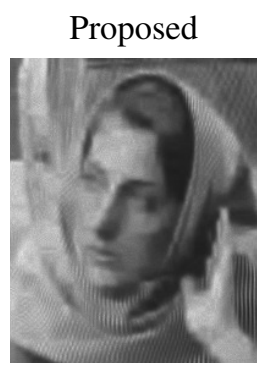

$23.99 \mathrm{~dB}$
Fig. 4. $\mathrm{SC}=$ Sparse Coding, $\mathrm{LER}=$ Low Error Rate. We observe that the proposed approach has a better PSNR and preseves well textures in comparison with the LER-SC.

speckling) [16] which is an acceleration of SAR-BM3D, 4) a sparsity-based approach: HKSVD (Homomorphic K-SVD). We observe that our method doesn't introduce artifacts in homogeneous areas as opposed to SAR-BM3D and FANS. Note also that our results do not show the artifact of isolated dark pixels as in HKSVD, which happens when the data-fidelity term is not adapted to SAR data. Finally our performance is comparable with PPB but doesn't suffer from the rare-patch effect.
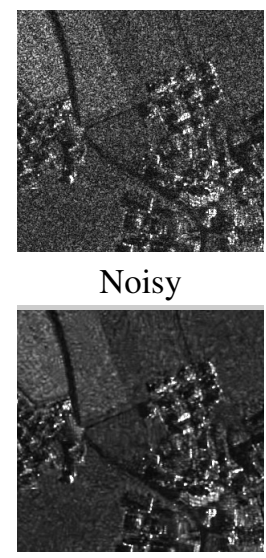

SAR-BM3D

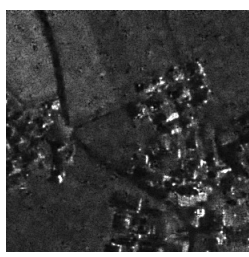

H-KSVD

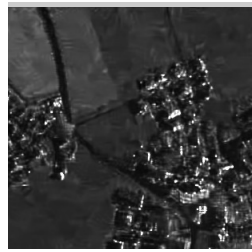

FANS

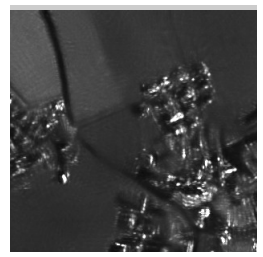

PPB

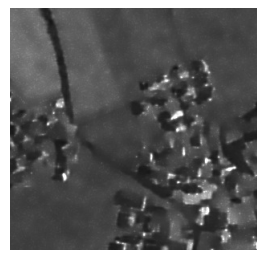

Proposed
Fig. 5. Comparison with other approaches (Rosen1 image).

\section{REFERENCES}

[1] J. W. Goodman, "Some fundamental properties of speckle," Journal of optical society of America, 1976.

[2] M. Aharon, M. Elad, and A.M. Bruckstein, "The K-SVD: An algorithm for designing of overcomplete dictionaries for sparse representation," IEEE Trans. on Signal Processing, vol. 54, no. 11, pp. 4311-4322, 2006.

[3] Y.C. Pati, R. Rezaiifar, and P.S. Krishnaprasad, "Orthogonal matching pursuit: recursive function approximation with applications to wavelet decomposition," in Proceedings of 27th
Asilomar Conference on Signals, Systems and Computers, Nov 1993, pp. 40-44 vol.1.

[4] S. Foucher, "SAR image filtering via learned dictionaries and sparse representations," in IEEE International Geoscience and Remote Sensing Symposium, IGARSS. IEEE, 2008, vol. 1, pp. I-229.

[5] P. Chainais, "Towards dictionary learning from images with non gaussian noise," in IEEE International Workshop on Machine Learning for Signal Processing (MLSP). IEEE, 2012, pp. $1-6$.

[6] T. Lu, S. Li, L. Fang, and J-A. Benediktsson, "SAR image despeckling via structural sparse representation," Sensing and Imaging, vol. 17, no. 1, pp. 1-20, 2016.

[7] D. Zoran and Y. Weiss, "From learning models of natural image patches to whole image restoration," in IEEE International Conference on Computer Vision (ICCV), Nov 2011, pp. 479486.

[8] S. Tabti, C-A. Deledalle, L. Denis, and F. Tupin, "Modeling the distribution of patches with shift-invariance: Application to SAR image restoration," in IEEE International Conference on Image Processing (ICIP), Oct 2014, pp. 96-100.

[9] J. Sulam and M. Elad, "Expected patch log likelihood with a sparse prior," in International Workshop on Energy Minimization Methods in Computer Vision and Pattern Recognition. Springer, 2015, pp. 99-111.

[10] H. Xie, L.E. Pierce, and F.T. Ulaby, "SAR speckle reduction using wavelet denoising and Markov random field modeling," IEEE Transactions on Geoscience and Remote Sensing, vol. 40, no. 10, pp. 2196-2212, Oct 2002.

[11] C-A. Deledalle, L. Denis, and F. Tupin, "Iterative weighted maximum likelihood denoising with probabilistic patch-based weights," IEEE Transactions on Image Processing, vol. 18, no. 12, pp. 2661-2672, 2009.

[12] A. Buades, B. Coll, and J.-M. Morel, "A non-local algorithm for image denoising," in IEEE Computer Society Conference on Computer Vision and Pattern Recognition, CVPR, June 2005, vol. 2, pp. 60-65 vol. 2.

[13] C-A. Deledalle, L. Denis, F. Tupin, A. Reigber, and M. Jäger, "NL-SAR: A unified non-local framework for resolutionpreserving (Pol)(In) SAR denoising," IEEE Transactions on Geoscience and Remote Sensing, vol. 53, no. 4, pp. 20212038, 2015.

[14] S. Parrilli, M. Poderico, C.V. Angelino, and L. Verdoliva, "A non-local SAR image denoising algorithm based on LLMMSE wavelet shrinkage," IEEE Transactions on Geoscience and Remote Sensing, vol. 50, no. 2, pp. 606-616, 2012.

[15] Kostadin Dabov, Alessandro Foi, Vladimir Katkovnik, and Karen Egiazarian, "Image denoising by sparse 3-D transformdomain collaborative filtering," IEEE Transactions on Image Processing, vol. 16, no. 8, pp. 2080-2095, 2007.

[16] D. Cozzolino, S. Parrilli, G. Scarpa, G. Poggi, and L. Verdoliva, "Fast adaptive nonlocal SAR despeckling," IEEE Geoscience and Remote Sensing Letters, vol. 11, no. 2, pp. 524528, 2014. 